\title{
Optimization dispatch rule of the Three Gorges Reservoir considering ecological and power generation benefit
}

\author{
Zhongzheng He ${ }^{1,2}$, Jianzhong Zhou ${ }^{1,2, *}$, Chengwei Lu ${ }^{1,2}$, Hui Qin ${ }^{1,2}$, Benjun Jia ${ }^{1,2}$, and Jingan Zhu ${ }^{1,2}$ \\ ${ }^{1}$ School of Hydropower and Information Engineering, Huazhong University of Science and Technology, Wuhan 430074, China \\ ${ }^{2}$ Hubei Key Laboratory of Digital Valley Science and Technology, Wuhan 430074, China
}

\begin{abstract}
This paper focus on optimization operation problem of ecology and power generation considering uncertainty of inflow on the Three Gorges reservoir. A model with constraint violation as objective is proposed to obtain ecological and power generation optimization dispatch rule of the Three Gorges Reservoir using stochastic dynamic programming. In the model, minimization of ecological water shortage and constraint violation and maximization of power generation benefit are taken as objective, simple discrete-time Markov chain and stochastic dynamic programming take into account the uncertainties of inflow. A case study shows that the proposed approach is feasible and efficient to produce a dispatch rule to balance ecological and economic benefits. Some conclusions of the influence of ecology and power generation are finally drawn.
\end{abstract}

\section{Introduction}

The Yangtze River is the third longest river in the world, the largest river in China. The Three Gorges Reservoir (TGR) is the key backbone of the Yangtze River Basin, and the operation and management of TGR will inevitably affect the river basin ecosystem of upper and lower reaches. Therefore, coordinating the power generation benefit and ecological impact has become an urgent problem remain to be studied.

In recent years, many researchers have carried out a series of studies around the issue of deterministic multiobjective optimization $[1,2]$, uncertain scheduling for power generation benefit $[3,4]$ and suitable ecological flow $[5,6]$. None of these studies involved the joint operation of ecological and power generation under uncertain water conditions. Based on the previous studies, this paper establishes the ecological and power generation multi-objective optimal dispatch model. The method of constraint violation as objective is proposed for constraint handling in the model. The simple discrete-time Markov chain and stochastic dynamic programming (SDP) take into account the uncertainties of inflow. In order to simplify the multi-objective stochastic dynamic programming problem, we choose constraint violation as the highest priority, and ecological and power generation are treated by linear fitness combination. Finally, the scheduling rules formulated by the proposed models and methods in paper are effective and reliable by testing in two cases.

\section{Mathematical model}

In order to establish a scheduling rule that takes into account both the benefits of ecology and power generation, a multi-objective optimal dispatching model with the objective of maximizing annual power generation and minimizing ecological water shortage is established. The details are as follows:

$$
\begin{gathered}
\max f_{1}=\max G=\sum_{t=1}^{T} N_{t} \Delta t \\
\min f_{2}=\min E=\sum_{t=1}^{T} \max \left(0, Q_{t}^{E}-Q_{t}\right) \Delta t
\end{gathered}
$$

where $G$ is the annual power generation, $N_{t}$ is the output in period $t, T$ is the total number of period, $\Delta t$ is the interval of period, $E$ is the annual ecological water shortage, $Q_{t}$ and $Q_{t}^{E}$ denote the outflow and suitable ecological flow of the downstream river. in period $t$, in period $t$, respectively. There are still some constraints to consider.

$$
\begin{gathered}
V_{t}=V_{t-1}+\left(I_{t}-Q_{t}\right) \cdot \Delta t \\
Z_{t}^{\min } \leq Z_{t} \leq Z_{t}^{\max } \\
\mid Z_{t}-Z_{t-1} \leq \Delta Z \\
Q_{t}^{\min } \leq Q_{t} \leq Q_{t}^{\max } \\
Z_{0}=Z_{\text {start }}, Z_{T}=Z_{\text {end }}
\end{gathered}
$$

where $V_{t}$ and $V_{t-1}$ is the water storage volume in period $t$ and $t-1$, respectively. $I_{t}$ is the inflow and outflow in period $t . Z_{t}^{\min }, Z_{t}^{\max }$ and $Z_{t}$ denote the maximum, minimum water level limits and current water level in period $t$, respectively. $Q_{t}^{\min }$ and $Q_{t}^{\max }$ denote the maximum and minimum water discharge limits in period $t$, respectively. $\Delta Z$ is maximum variation amplitude of water level. $Z_{\text {start }}$ and $Z_{\text {end }}$ denote initial water level and terminal water level, respectively.

\section{Stochastic Dynamic Programming}

Because of the inflow uncertainty, the optimal scheduling of TGR is a stochastic optimal control problem. Therefore,

\footnotetext{
${ }^{*}$ Corresponding author: jz.zhou@mail.hust.edu.cn
} 
we construct a scheduling rule to consider inflow uncertainty using stochastic dynamic programming. The simple discrete-time Markov chain is adopted to describe the inflow. Assume that the probability distribution of the next state depends only on the current state, not on the state in the previous step. Then inflow value is divided into several intervals $I_{1}, I_{2}, I_{3}, \ldots, I_{N}$ and one-step transition probability matrices are shown as follow:

$$
\begin{gathered}
P\left(I_{t+1} \mid I_{t}, I_{t-1}, \ldots, I_{1}\right)=P\left(I_{t+1} \mid I_{t}\right) \\
{\left[\begin{array}{cccc}
p_{11} & p_{12} & \cdots & p_{1 n} \\
p_{21} & p_{22} & \cdots & p_{2 n} \\
\vdots & \vdots & \ddots & \vdots \\
p_{n 1} & p_{n 2} & \cdots & p_{n n}
\end{array}\right]}
\end{gathered}
$$

Where $P\left(I_{t+1} \mid I_{t}\right)$ stands for one-step transition probability, $\quad p_{i j}=P\left(I_{t+1}=q_{j} \mid I_{t}=q_{i}\right) \quad$ denotes the probability when the inflow value of period $t$ is $q_{i}$ and the value of period $t+1$ is $q_{j}$.

The optimal scheduling rule of TGR can be considered as a multistage Markov decision process when stochastic inflows is described as a simple discrete-time Markov chain. Stochastic dynamic programming is an effective way to solve multistage Markov decision process problems. According to the future inflow forecast in each period, we make corresponding optimal decision with water level as the state variable. The recursion equation of SDP can be described as formula (10)

$$
\begin{aligned}
& R_{t}\left(I_{t}, V_{t-1}, \mathrm{Z}_{t}\right)=\max _{\mathrm{Z}_{t} \in \Omega_{\mathrm{t}}}\left[B_{t}\left(V_{t-1}, I_{t}, \mathrm{Z}_{t}\right)\right. \\
&\left.+\sum_{n=1}^{N} P\left(I_{t+1} \mid I_{t}\right) R_{t+1}\left(I_{t+1}, V_{t}, \mathrm{Z}_{t+1}\right)\right] \\
&(t=1,2, \ldots, \mathrm{T}-1 ; n=1,2, \ldots, N)
\end{aligned}
$$

Where $R_{t}\left(I_{t}, V_{t-1}, Z_{t}\right)$ denotes optimal remaining benefit function, it represents optimal total expected benefit from $t$ to $T$ period with optimal decision in $t$ period when the initial reservoir capacity is $V_{t}$ and inflow is $I_{t}$.

The optimal dispatching rules of the Three Gorges reservoir can be obtained by the iteration of the recursive equation in formula (10). The optimal dispatching rules can be described below.

$$
\left\{\begin{array}{l}
R_{t}=R_{t}\left(I_{t}, V_{t-1}, Z_{t}\right) \\
Z_{t}=Z_{t}\left(t, I_{t}, V_{t-1}\right)
\end{array}\right.
$$

\section{Implementation}

It need processing methods for constraints and multiobjective optimization that stochastic dynamic programming is applied to obtain optimization dispatch rule of the TGR considering ecological and power generation benefit under the condition of inflow uncertainty. Constraint conditions are usually handled by penalty function, constraint violation as objective and $\varepsilon$ constraint method. The optimization models mentioned in this paper mainly involve discrete state and multiobjective optimization, so constraint violation as objective is chosen for constraint handling.
In general, the constraint of constrained optimization problems can be mathematically formulated as follows:

$$
\text { subject to } \begin{cases}g_{i}(\overrightarrow{\mathrm{x}}) \leq 0 & i=1,2, \cdots, \mathrm{m} \\ h_{j}(\overrightarrow{\mathrm{x}})=0 & j=1,2, \cdots, \mathrm{n}\end{cases}
$$

where $g_{i}(\overrightarrow{\mathrm{x}})$ is inequality constraints, $\mathrm{m}$ is the total number of inequality constraint. $h_{j}(\overrightarrow{\mathrm{x}})$ is equality constraints and $\mathrm{n}$ is the total number of equality constraints. The value of constraint violation $\varphi(\overrightarrow{\mathrm{x}})$ can be calculated in the following formula (11).

$$
\varphi(\overrightarrow{\mathrm{x}})=\sum_{i=0}^{m} \max \left(0, g_{i}(\overrightarrow{\mathrm{x}})\right)+\sum_{j=1}^{n}\left(\left|h_{j}(\overrightarrow{\mathrm{x}})\right|, 0\right)
$$

In addition, in order to unify the dimensions of different physical quantities, the violation of all equality and inequality constraints will eventually be converted to constraint violation of water volume. The constraint violation of water volume is regarded as the third objective of the optimization model to measure the degree of constraint violation of different decisions as below.

$$
\begin{aligned}
\min f_{3} & =\min C=\sum_{t=1}^{T} \varphi\left(V_{t-1}, I_{t}, \mathrm{Z}_{t}\right) \\
& =\sum_{t=1}^{T} \sum_{i=0}^{m}\left[\max \left(0, g_{i}\left(V_{t-1}, I_{t}, \mathrm{Z}_{t}\right)\right)\right. \\
& \left.+\sum_{j=1}^{n}\left(\left|h_{j}\left(V_{t-1}, I_{t}, \mathrm{Z}_{t}\right)\right|, 0\right)\right]
\end{aligned}
$$

where $C$ is the constraint violation of water volume. The decision is infeasible solution when $C>0$.

The multi-objective stochastic dynamic programming is difficult to solve. To simplify the problem, we choose $C$ as the highest priority, and $E$ and $G$ are treated by linear fitness combination. After calculating the optimal expected benefit of all possible decisions in the current state, $C, E$ and $G$ can be converted into $C^{\prime}, E^{\prime}$ and $G^{\prime}$ respectively by standard $0-1$ transformation. This process can be described as follows.

$$
\begin{gathered}
{\left[\begin{array}{ccc}
C_{t, 1} & G_{t, 1} & E_{t, 1} \\
C_{t, 2} & G_{t, 2} & E_{t, 2} \\
\vdots & \\
C_{t, M} & G_{t, M} & E_{t, M}
\end{array}\right]} \\
A^{\prime}=\frac{A-A_{\min }}{A_{\max }-A_{\min }} \\
B^{\prime}=\frac{B_{\max }-B}{B_{\max }-B_{\min }}
\end{gathered}
$$

Where formula (10) represents optimal expected benefit matrix of all possible decisions for current state in $t$ period. The formula (16) and formula (17) represents the benefit attribute and cost attribute respectively of standard 0-1 transformation. $C$ and $E$ belong to the cost attribute using formula (17); $G$ belongs to the benefit attribute using formula (16). Then the three objectives can be assigned different weights so that they can be combined into one goal.

$$
\max f=\max F=\alpha C^{\prime}+\left[\beta G^{\prime}+(1-\beta) E^{\prime}\right]
$$

Where $F$ is weighted combined objective function, $\alpha$, $\beta$ and $(1-\beta)$ stand for the weight for $C^{\prime}, G^{\prime}$ and $E^{\prime}$ respectively. In order to ensure highest priority of $C^{\prime}, \alpha$ needs to be set large enough to be 3 as $G^{\prime}, E^{\prime}, \beta \in[0,1]$ 
and $\left[\beta G^{\prime}+(1-\beta) E^{\prime}\right] \leq 2$. In this way, the optimal dispatching rules for coordinating ecological and power generation benefits can be obtained under the conditions of priority ensuring the feasibility of decision-making and the uncertainty of inflow. In addition, $F$ is only used to generate the decision stage of scheduling rules, and $\mathrm{R}$ stores the optimal expected benefits from $t$ to $T$ period seeing as formula (18).

$$
R_{t}\left(I_{t}, V_{t-1}, \mathrm{Z}_{t}\right)=\left[\begin{array}{lll}
C_{t} & G_{t} & E_{t}
\end{array}\right]
$$

\section{Case Study}

The Three Gorges Reservoir (TGR), located in Yichang, Hubei province, China. It plays a key role in water resources management and ecological environment protection in the Yangtze River Basin. The basic parameters of TGR is shown in Table 1.

Table 1. The basic parameters of TGR

\begin{tabular}{|c|c|}
\hline Total capacity $\left(10^{8} \mathrm{~m}^{3}\right)$ & 393 \\
\hline Installed capacity $(\mathrm{MW})$ & 22400 \\
\hline Normal Water Level $(\mathrm{m})$ & 175 \\
\hline Dead Water Level $(\mathrm{m})$ & 145 \\
\hline Upriver water level range $(\mathrm{m})$ & {$[175,145]$} \\
\hline discharge range $\left(\mathrm{m}^{3} / \mathrm{s}\right)$ & {$[99800,6000]$} \\
\hline
\end{tabular}

Considering the water requirement of various ecological factors, the suitable ecological discharge for the lower reaches of the Three Gorges was proposed in Ref [1] as follows.

Table 2. The suitable ecological flow of the TGR $\left(\mathrm{m}^{3} / \mathrm{s}\right)$

\begin{tabular}{|c|c|c|c|c|c|c|}
\hline month & 1 & 2 & 3 & 4 & 5 & 6 \\
\hline$Q_{t}^{E}$ & 5500 & 5500 & 5500 & 11000 & 11884 & 13570 \\
\hline month & 7 & 8 & 9 & 10 & 11 & 12 \\
\hline$Q_{t}^{E}$ & 22240 & 19845 & 18143 & 19593 & 12000 & 5970 \\
\hline
\end{tabular}

The one-step transition probability matrices will be constructed using the method mentioned in Section 3 based on historical runoff data from 1883 to 2011, and the time scale of scheduling is month. As space is limited, the detailed content won't be described here

Case 1 test the constraint violation objectification for constraint handling. The ecological benefit are not considered for simplification with $\beta=1$. The one-step transition probability matrices are as input, then the optimization dispatch rule of the TGR considering power generation benefit with constraint violation as objective (Rule A) and without constraint violation as objective (Rule B) can be obtained using SDP. The simulation results of the two dispatching rules from 1883 to 2011 are shown in the figure.

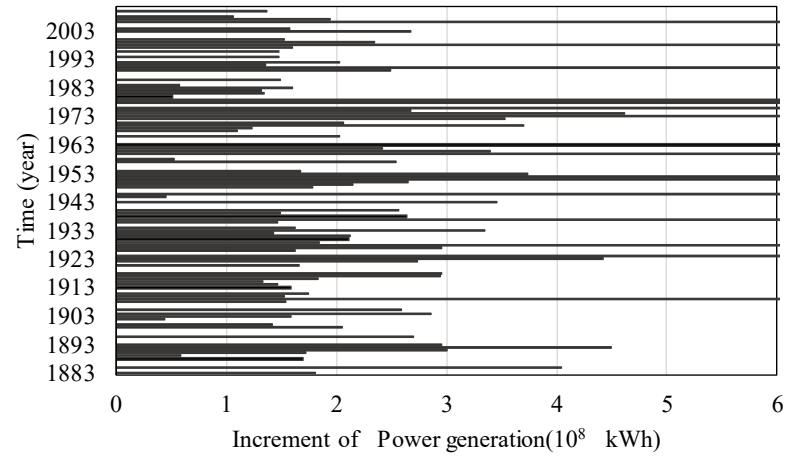

Figure 1. The simulation results from 1883 to 2011.

In Figure 1, Increment of Power generation represents increased power generation using Rule A than using Rule B. And it means that TGR cannot simulate the operation using the rule $\mathrm{B}$ when the increment of power generation reaches 6 . There are 16 years that TGR could not schedule using the scheduling rule B from 1883 to 2011 . The main reason is that the minimum discharge of TGR is $6000 \mathrm{~m}^{3} / \mathrm{s}$, the rule $\mathrm{B}$ makes it have limitations for satisfying all inflow conditions without the relaxation constraints of constraint violation as objective. In addition, it is obvious that the power generation using Rule A is larger than Rule B. These results show that the method of constraint violation as objective can effectively handle multiple constraints and increase benefits through relaxation constraints.

Case 1 validates the effectiveness of the model and method proposed in this paper. Coordination of ecological and power generation benefits will be concerned in Case 2. The contrast experiment is designed with the weight $\beta$ $\in[0,1]$ and the step is 0.2 in the process of generating scheduling rules. The control group was the result with control parameter $\beta=1$, which only considers power generation benefits in this scheduling rule. The results of the 6 set of scheduling rules are shown below.

Table 3. The average power generation and ecological water shortage using the 6 set of scheduling rules from 1883 to 2011

\begin{tabular}{|c|c|c|c|c|}
\hline$\beta$ & $\begin{array}{c}\text { ecological } \\
\text { water } \\
\text { shortage } \\
\left(10^{8} \mathrm{~m}^{3}\right)\end{array}$ & $\begin{array}{c}\text { Percentage } \\
\text { Compared } \\
\text { to } \beta=1\end{array}$ & $\begin{array}{c}\text { power } \\
\text { generation } \\
\left(10^{8} \mathrm{kWh}\right)\end{array}$ & $\begin{array}{c}\text { Percentage } \\
\text { Compared } \\
\text { to } \beta=1\end{array}$ \\
\hline 1 & 250.70 & $100.00 \%$ & 970.60 & $100.00 \%$ \\
\hline 0.8 & 250.47 & $99.91 \%$ & 970.53 & $99.99 \%$ \\
\hline 0.6 & 249.50 & $99.52 \%$ & 967.98 & $99.73 \%$ \\
\hline 0.4 & 249.95 & $99.70 \%$ & 961.89 & $99.10 \%$ \\
\hline 0.2 & 249.95 & $99.70 \%$ & 961.84 & $99.10 \%$ \\
\hline 0 & 249.67 & $99.59 \%$ & 961.90 & $99.10 \%$ \\
\hline
\end{tabular}

Table 3 shows that power generation and ecological water shortage decrease with $\beta$ becoming smaller. This shows that the objectives of power generation and ecological water shortage are in conflict, because power generation is in pursuit of maximum and ecological water shortage is in pursuit of minimum, and there is competitive water use between them. However, the reduction of power generation and ecological water shortage is not monotonous with $\beta$ decreasing. We selected the simulation process of 2008 using the scheduling rules with the weight $\beta \in[0,1]$ and the step is 0.1 in the process of generating scheduling rules for 
analysis. The result and simulation process are shown in the table and figure as below.

Table 3. The power generation and ecological water shortage using scheduling rules generated with different $\beta$ in 2008

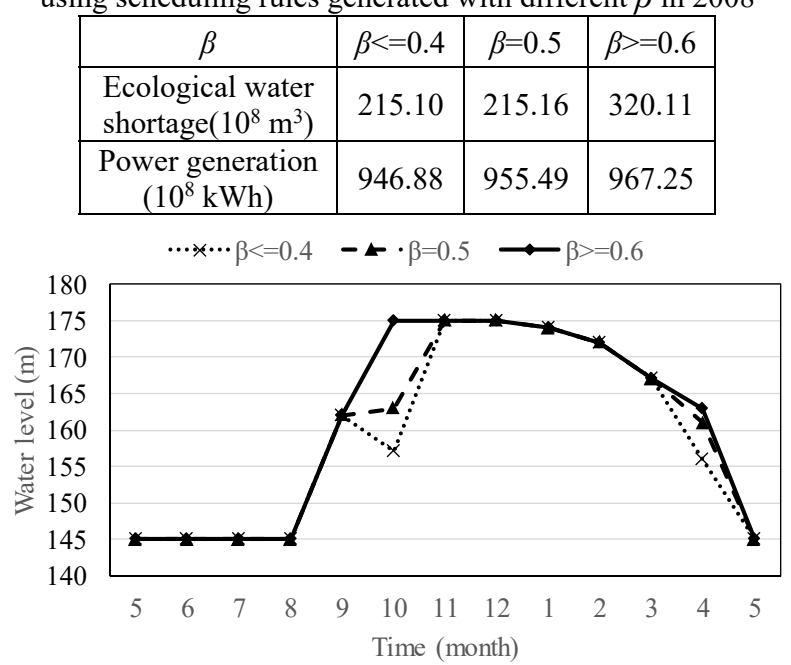

(a) Water level

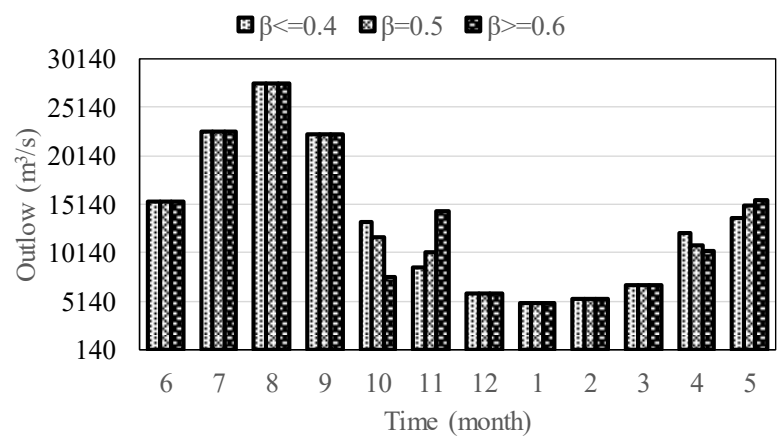

(b) Outflow

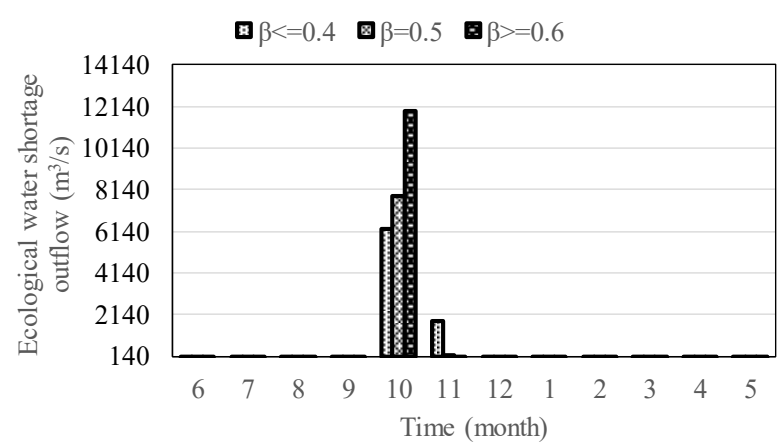

(c) Ecological water shortage outlow

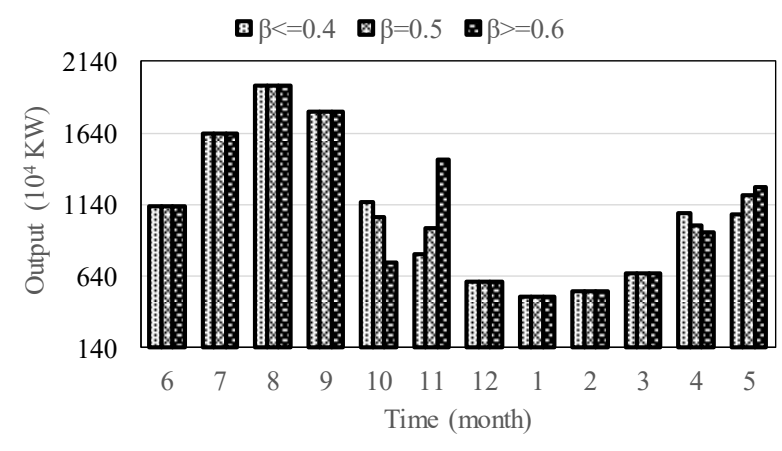

(d) Output

Figure 2. The simulation results in 2008 with different $\beta$
$R_{t}=R_{t}\left(I_{t}, V_{t-1}, \mathrm{Z}_{t}\right)$ and $\mathrm{Z}_{t}=\mathrm{Z}_{t}\left(t, I_{t}, V_{t-1}\right)$ show that the optimal decision is related to the initial storage capacity and the inflow at the current stage. Only part of the scheduling rules can be seen in Figure 2, because the type of inflow is 2008. According to the type of inflow in 2008 , there are three kinds of dispatching rules based on $\beta \leq 0.4, \beta=0.5$ and $\beta \geq 0.6$. From Figure 2 (a), It can be seen that the larger $\beta$, the greedier the dispatching rules are in pursuit of power generation, thus the higher the water level process specified by the dispatching rules. The ecological water shortage mainly appeared in October in Figure 2 (c). Combining with Figure 2 (a), (b) and (c), it can be seen that the regulation focuses on ecological benefits when $\beta$ is small, so TGR increased the discharge and reduced the ecological water shortage in October under the guidance of dispatching rules. All of these show that the scheduling rules formulated by the proposed models and methods in paper are effective and reliable.

\section{Conclusions}

Focus on ecological and power generation optimization operation problem of the TGR considering uncertainty of inflow. A model is proposed to obtain ecological and power generation optimization dispatch rule of the Three Gorges Reservoir using stochastic dynamic programming. In addition, the method of constraint violation as objective is proposed for constraint handling in the model. For multi-objective stochastic dynamic programming problem, we choose constraint violation as the highest priority, and ecological and power generation are treated by linear fitness combination. Finally, the scheduling rules formulated by the proposed models and methods in paper are tested in two cases, the results shows that the method of constraint violation as objective can effectively handle multiple constraints and increase benefits through relaxation constraints, and scheduling rules formulated by the proposed models and methods in paper are effective and reliable. But there are still some problems, such as that the one-step transition probability matrices description inflow characteristics are not accurate enough, and how to achieve non dominated multi-objective optimization in stochastic programming instead of linear weighting, etc. These are the focus of future research.

\section{Acknowledgments}

This work is supported by the National Natural Science Foundation Key Project of China (No. 91547208), the National Natural Science Foundation of China (No. 51579107, No. 91647114, and No. 51709119), the National Key R\&D Program of China (No. 2016YFC0402205) and the Foundation of Huazhong University of Science and Technology (No. 2017KFYXJJ209).

\section{References}

1. X. Wang, J. Zhou, C. Wang, International Journal of Modeling \& Optimization 3, 241-245 (2015) 
2. C. Wang, J. Zhou, X. Wang, et al, International Conference on Control, Automation and Robotics. IEEE 204-208 (2015)

3. L. Yuan, J. Zhou, C. Li, et al. Water Resources Management 10, 3347-3361 (2006)

4. J. Zhou, M. Xie, Z. He, et al, Water Resources Management 11, 3543-3555 (2017)

5. Z. R. Pan, X-H. R, X. U. Jing, Journal of Hydraulic Engineering, 1, 119-126 (2013)

6. J. Li, Z. Q. Xia, G. H. Ma, et al, Acta Ecologica Sinica, (2007) 